\title{
Superficial Thrombophlebitis
}

National Cancer Institute

\section{Source}

National Cancer Institute. Superficial Thrombophlebitis. NCI Thesaurus. Code C78634.

Thrombosis and inflammation of a superficial vein of the extremities. Symptoms include red discoloration and warmth of the skin and pain along the affected vein. 